\title{
Regulation by Protein Kinase A Switching of Axonal Pathfinding of Zebrafish Olfactory Sensory Neurons through the Olfactory Placode-Olfactory Bulb Boundary
}

\author{
Tomoyuki Yoshida, Aya Ito, Naoto Matsuda, and Masayoshi Mishina \\ Department of Molecular Neurobiology and Pharmacology, Graduate School of Medicine, University of Tokyo, and \\ SORST, Japan Science and Technology Corporation, Tokyo 113-0033, Japan
}

\begin{abstract}
Cumulative evidence suggests that neural network formation requires an ingenious regulation of the attractive and repulsive responses of growing axons to guidance cues. We examined the role of intracellular protein kinase $A(P K A)$ signaling in the axonal pathfinding of olfactory sensory neurons in transparent zebrafish embryos. Microinjection of an olfactory marker protein gene promoter-driven double-cassette vector directed the expression of both the dominant form of PKA and green fluorescent protein fused with the microtubule-associated protein tau in the same olfactory neurons. The dominant-negative form of PKA enhanced the turning of olfactory neuron axons in the olfactory placode, whereas the disturbance effect of the con-
\end{abstract}

stitutively active form on the axonal pathfinding was prominent in the olfactory bulb. Consistently, forskolin treatment severely inhibited the axonal extension in the olfactory bulb, but not in the olfactory placode. These results suggest that the switching of PKA signaling in developing olfactory sensory neurons is important for axonal pathfinding through the boundary between the olfactory placode and the olfactory bulb in vivo. We thus propose that the regulation of PKA signaling plays a key role in the longdistance axonal pathfinding through intermediate guideposts.

Key words: protein kinase A; axon guidance; olfactory sensory neuron; zebrafish; double-cassette vector; olfactory marker protein; olfactory placode; olfactory bulb
Elucidation of how the neural network is formed and modulated is essential to understanding how the brain functions. The initial steps of neural wiring are axonal pathfinding, target selection, and synapse formation. Attractive and repulsive guidance molecules play key roles in axonal pathfinding (Tessier-Lavigne and Goodman, 1996; Mueller, 1999). These extracellular guidance molecules interact with receptors on the neural growth cone to determine the direction of axon extension. Simple attraction and repulsion, however, cannot explain the long-distance axon guidance where extending axons should be attracted toward intermediate guideposts and have to leave when they reach there. Shirasaki et al. (1998) showed that rat metencephalon commissural axons lost their responsiveness to the floor plate-derived chemoattractant, Netrin, once they crossed the floor plate in vitro. Netrin and Slit mediate attractive and repulsive guidance of commissural axons at the midline in Drosophila, respectively (Harris et al., 1996; Mitchell et al., 1996; Kidd et al., 1999). The expression of the Slit receptor, Roundabout (Robo), in commissural axons is low before midline crossing but is strongly upregulated after crossing (Kidd et al., 1998, 1999). In cultured Xenopus spinal neurons, the attractive responses to Netrin mediated by the Deleted in Colorectal Cancer Netrin receptor were converted to

\footnotetext{
Received July 9, 2001; revised April 1, 2002; accepted April 4, 2002.

This work was supported in part by research grants from the Japan Science and Technology Corporation and the Ministry of Education, Culture, Sports, Science, and Technology of Japan. T. Y. was a recipient of the Fellowship for Young Scientists from the Japan Society for the Promotion of Science. We are grateful to Y. Ishida for data processing and K. Kinomoto for help with zebrafish breeding. We thank Dr. G. S. McKnight for plasmids pCQ87/R196 and pMT-Rev ${ }^{\mathrm{AB}}$-neo and Dr. P. Mombaerts for the IRES-tauGFP-LNL cassette.

Correspondence should be addressed to Masayoshi Mishina, Department of Molecular Neurobiology and Pharmacology, Graduate School of Medicine, University of Tokyo, Hongo 7-3-1, Bunkyo-ku, Tokyo 113-0033, Japan. E-mail: mishina@m.u-tokyo.ac.jp.
}

Copyright (ㄷ) 2002 Society for Neuroscience $\quad 0270-6474 / 02 / 224964-09 \$ 15.00 / 0$ repulsive ones by the exogenous expression of the UNC-5 Netrin receptor (Hong et al., 1999). The exogenous expression of Robo silenced the attractive response to Netrin (Stein and TessierLavigne, 2001). Thus, there should be an ingenious regulatory mechanism for the responsiveness of growing axon terminals toward guidance cues. One of the possible regulatory mechanisms might be protein kinase A (PKA) signaling, because in vitro pharmacological manipulations lowering the intracellular levels of cAMP or PKA activity changed the attractive response to Netrin or brain-derived neurotrophic factor (BDNF) into a repulsive response in cultured Xenopus spinal neurons (Ming et al., 1997; Song et al., 1997). However, it remains unknown whether PKA signaling actually changes in vivo and regulates the axonal extension through intermediate guideposts. To address this issue, we developed a novel strategy that enables visualization and manipulation of developing neurons in vivo. Olfactory systems in transparent zebrafish embryos are suitable to analyze axonal pathfinding in vivo (Hansen and Zeiske, 1993; Dynes and Ngai, 1998; Whitlock and Westerfield, 1998). We analyzed the effects of constitutively active and dominant-negative forms of PKA on the axonal pathfinding of olfactory sensory neurons in living zebrafish embryos using olfactory marker protein (Omp) gene promoter-driven effector-reporter double-cassette vectors. Modulations of PKA affected the turning behavior of developing axons of olfactory sensory neurons in a region-specific manner. These results suggest that switching of PKA signaling is important for axonal pathfinding of zebrafish olfactory sensory neurons through the boundary from the olfactory placode to the olfactory bulb in vivo.

\section{MATERIALS AND METHODS}

Animals. The zebrafish $\mathrm{AB}$ strain was used. Zebrafish embryos were raised at $28^{\circ} \mathrm{C}$ in embryo medium (EM) containing $17 \mathrm{~mm} \mathrm{NaCl}, 0.27 \mathrm{~mm}$ $\mathrm{CaCl}_{2}, 0.66 \mathrm{~mm} \mathrm{MgSO}_{4}, 0.4 \mathrm{~mm} \mathrm{KCl}$, and $1 \times 10^{-5} \%$ methylene blue. 
Cloning of the zebrafish omp gene and construction of olfactory neuronspecific expression vectors. The $0.17 \mathrm{~kb}$ DNA fragment of the zebrafish omp gene was isolated by PCR with degenerate primers designed based on the coding sequence of the human, rodent, and frog omp genes (Buiakova et al., 1994; Rössler et al., 1998): 5'-CTGCAGCA(A/G)(A/ C)A(C/T)TGGACNCCNGA-3' and 5'-CTGCAG(A/G)AA(A/G)TACATNAC(C/T)TT-3' (N, four nucleotides). By screening a zebrafish bacterial artificial chromosome library (Incyte Genomics, St. Louis, MO) using the PCR fragment as a probe, we isolated the entire omp gene. (The nucleotide and deduced amino acid sequences can be found in the public database as accession number AB073551.) Alignment of amino acid sequences was performed using GENETYX-MAC 9.0 software (Software Development, Tokyo, Japan). The $6.2 \mathrm{~kb}$ BglII fragment contained the $0.5 \mathrm{~kb}$ omp coding sequence without the intron, $2.7 \mathrm{~kb}$ of $5^{\prime}$ upstream sequence, and $3.0 \mathrm{~kb}$ of $3^{\prime}$ downstream sequence. The omp coding region of the $6.2 \mathrm{~kb} B g l \mathrm{II}$ fragment was replaced by $2.0 \mathrm{~kb}$ tau-enhanced green fluorescent protein (EGFP), $2.0 \mathrm{~kb}$ tau-red fluorescent protein from Discosoma $s p$. (DsRed), $1.1 \mathrm{~kb}$ of the constitutively active form of PKA (PKA*), and $1.1 \mathrm{~kb}$ of the dominant-negative form of PKA (dnPKA) coding sequences in Pomp-GFP, Pomp-DsRed, Pomp-

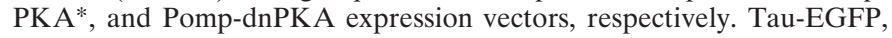
DsRed, PKA*, and dnPKA coding sequences were obtained from the IRES-tauGFP-LNL cassette (provided by Dr. P. Mombaerts, Rockefeller University, New York, NY), pDsRed-N1 (Clontech, Palo Alto, CA), and pCQ87/R196 and pMT-Rev ${ }^{\mathrm{AB}}$-neo (provided by Dr. G. S. McKnight, University of Washington, Seattle, WA), respectively. The 0.7 $\mathrm{kb}$ HindIII-BspLU11I fragment of the 5' upstream sequence of the omp gene, the tau-EGFP coding sequence, and the $0.45 \mathrm{~kb}$ EcoRI-Bam HI fragment carrying simian virus 40 (SV40) poly(A) additional signal sequence from $\mathrm{pSV}$ - $\beta$-galactosidase control vector (Promega, Madison, WI) were ligated to yield the control GFP expression vector. PompDsRed, Pomp-PKA*, and Pomp-dnPKA were joined with the GFP expression vector in a tail-to-tail manner to yield double-cassette expression vectors, DsRed-GFP, PKA*-GFP, and dnPKA-GFP, respectively.

In situ hybridization. The $0.8 \mathrm{~kb}$ zebrafish omp cDNA carrying the coding and $3^{\prime}$ untranslated regions was amplified by RT-PCR using 5'-CAACATGTCTCTGGAGTTGACG-3' and 5'-GCGTTATAACTCCCTTAAAGTCTC- $3^{\prime}$ as primers and subcloned into the pBluescript II SK + (Stratagene, La Jolla, CA) to yield pBSK-OMPC3. Digoxigenin (DIG)-11-UTP-labeled sense and antisense RNA probes specific for the zebrafish omp gene were synthesized in vitro with T7 and T3 RNA polymerases using NotI- and SalI-cleaved pBSK-OMPC3, respectively, as described in the instruction manual of a DIG RNA Labeling Kit (Roche, Indianapolis, IN). In situ hybridization, antibody staining, paraffinembedding, and sectioning of whole embryos were performed as described previously (Mori et al., 1994; Jowett, 1999). Deparaffinized sections were counterstained with $0.5 \%$ methyl green for $10 \mathrm{~min}$, washed with water and 1-butanol three times, respectively, dehydrated with xylene three times, and mounted in malinol for microscopy.

Microinjection of DNA into zebrafish embryos and generation of transgenic lines. Expression vectors linearized by SalI were dissolved in 100 mM KCl containing $0.05 \%$ phenol red. Approximately $0.2-0.5 \mathrm{nl}$ of the DNA solution at a concentration of $50-100 \mathrm{ng} / \mu \mathrm{l}$ was injected into the cytoplasm of the 1- to 4-cell embryos.

For the generation of stable lines, 1-cell embryos were injected with Pomp-GFP. The injected embryos exhibiting tau-EGFP fluorescence in the olfactory placode were maintained to sexual maturity and crossed with wild-type fish to examine the transmission of the transgene to the next generation. We obtained two transgenic lines stably expressing tau-EGFP selectively in the olfactory sensory neurons from 228 injected embryos.

Forskolin treatment. The tau-EGFP transgenic embryos at $18 \mathrm{hr}$ postfertilization (hpf) were dechorionated and soaked in EM containing $0.05 \%$ ethanol with or without $50 \mu \mathrm{M}$ forskolin. The treated embryos were mounted in $1 \%$ low-melting point agarose in EM at 22, 28, and 40 hpf for microscopy.

Microscopy. Zebrafish embryos expressing tau-EGFP in olfactory sensory neurons were screened by a fluorescent microscope using NIBA filter (Olympus Optical, Tokyo, Japan) at 28-29 hpf. Screened embryos were then mounted in $1 \%$ low-melting point agarose in EM at $50 \mathrm{hpf}$. DiI labeling of embryos was performed as described by Dynes and Ngai (1998). Embryos were imaged by a Bio-Rad (Hercules, CA) MRC-1024 confocal microscope with argon and/or helium-neon lasers using an oil-immersion Planapo $60 \times$ objective lens (Olympus Optical).

Image processing. Stacks of $512 \times 512$ pixel images were reconstructed three-dimensionally using NIH Image 1.62 software. The origin was set at the nasal pit (i.e., the point at which the dendrites of olfactory neurons came together). Distance from the origin was used to indicate the position of each flection. By connecting flections on the axons in the three-dimensional images that were reconstructed, axon trajectories were traced. Axons were traced from the axon hillock to the terminal if possible, but axons whose soma could not be assigned were also included in the data. For simplicity, minor branches were ignored and only major axons giving clear fluorescence were traced.

Parameters. Terminal reach, maximal reach, and complexity values were compared between each group of axons. Terminal reach is the distance from the center of the nasal cavity (origin) to the axon terminal. Maximal reach is the distance from the origin to the farthest point on the axonal trajectory. Complexity is the summation of angle made by the original direction and new direction per length of axon. Regional complexity was calculated by the summation of turning angles per axon length within an area.

\section{RESULTS}

\section{Visualization of developing zebrafish olfactory neurons in vivo}

Olfactory marker protein is selectively expressed in mammalian olfactory sensory neurons (Farbman and Margolis, 1980). We isolated the zebrafish omp gene. The deduced 155 aa sequence of zebrafish Omp shares 56, 51, and 53\% identities with Xenopus, mouse, and human OMPs (Buiakova et al., 1994; Rössler et al., 1998), respectively (Fig. $1 A$ ). In situ hybridization analysis showed that the zebrafish omp gene was selectively expressed in the olfactory placode of $28 \mathrm{hpf}$ embryos (Fig. 1B,C). Almost all of the cells proximal to the nasal pit in the apical region of the olfactory placode expressed the $\mathrm{omp}$ gene at $28 \mathrm{hpf}$ (Fig. 1D), where odorant receptor genes were expressed (Whitlock and Westerfield, 1998). To label the olfactory sensory neurons, we expressed EGFP fused with the microtubule-associated protein tau (Brand, 1995) by using the $2.7 \mathrm{~kb} \mathrm{5'}$ upstream and $3.0 \mathrm{~kb} \mathrm{3}$ downstream sequences of the zebrafish omp gene (Fig. 2A). Microinjection of the tau-EGFP construct into zebrafish embryos resulted in the selective labeling of olfactory sensory neurons including their axons and dendrites.

To visualize the development of entire olfactory neurons in living zebrafish embryos, we isolated two transgenic zebrafish lines carrying the omp promoter-driven tau-EGFP fusion gene (Fig. 2B). The fluorescence of the tau-EGFP fusion protein in these lines first appeared in the olfactory placode at $22 \mathrm{hpf}$ (Fig. $2 C)$. Several labeled cells were monopolar neurons sprouting their axons. The monopolar neurons with round cell bodies may be the olfactory pioneer neurons described by Whitlock and Westerfield (1998). Bipolar olfactory sensory neurons with dendrites and axons appeared by $28 \mathrm{hpf}$. Figure $2 D$ shows tens of fluorescent-labeled olfactory neurons in zebrafish embryos at 28 hpf. Olfactory sensory neurons were arrayed in a circle and extended their short and thick dendrites toward the nasal pit at the center of the circle. The bipolar olfactory neurons projected their axons toward the developing telencephalon, and their axon terminals were mainly around the boundary between the olfactory placode and the developing olfactory bulb.

There were hundreds of olfactory neurons at $50 \mathrm{hpf}$ (Fig. 2E). Their dendrites became longer and thinner. The axons fasciculated to form the olfactory nerve and crossed the boundary between the olfactory placode and the olfactory bulb (BPB) through a restricted region, as described by Hansen and Zeiske (1993). Almost all of the olfactory neurons extended their axons through the boundary into the developing olfactory bulb by 50 hpf. After crossing the boundary, the axons defasciculated in the olfactory bulb. 


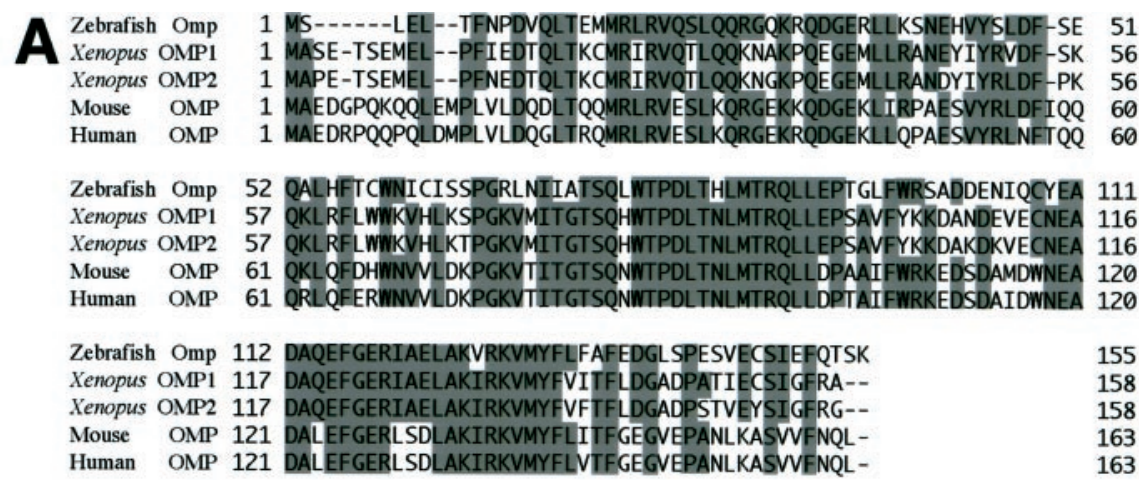

Figure 1. Deduced amino acid sequence and expression of zebrafish Omp. A, Alignment of the amino acid sequences of zebrafish, Xenopus, mouse, and human olfactory marker proteins. Amino acid residues identical in three or more sequences are shaded. Dashes represent gaps in the alignment. $B$, $C$, Dorsal views of the head of $28 \mathrm{hpf}$ embryos stained by in situ hybridization with DIG-labeled sense $(B)$ and antisense $(C)$ probes of the zebrafish omp mRNA. Scale bar, $100 \mu \mathrm{m}$. $D$, A paraffin section of the olfactory placode stained by in situ hybridization with the antisense probe of the zebrafish omp mRNA. The section was counterstained with methyl green. $D$ corresponds to the inset in $C . O B$, Olfactory bulb; $O P$, olfactory placode; $E Y E$, eye. The arrowhead in $D$ points to the nasal pit.
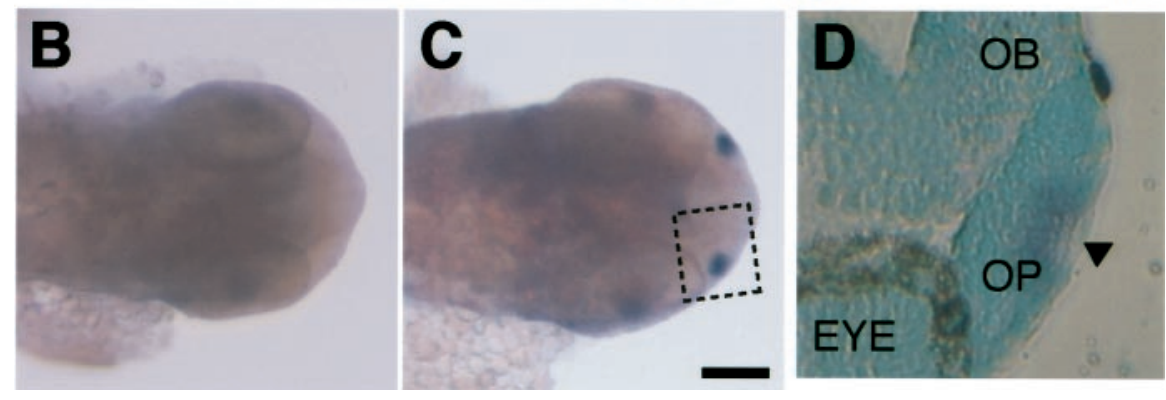

At $72 \mathrm{hpf}$, the nasal pit became wide and the olfactory nerve fasciculated more tightly at the BPB (Fig. $2 F$ ). The boundary formed the ethmoid structure (http://zdb.wehi.edu.au:8282/ zf_info/anatomy.html), and bundles of olfactory axons crossed this structure through the slit of the basal lamina of the olfactory placode (Hansen and Zeiske, 1993). In the olfactory bulb, the olfactory nerves defasciculated and branched out to several zones in accordance with previous observations using DiI and BODIPY labeling (Dynes and Ngai, 1998). The increases in the number of olfactory neurons and the extent of axonal extension between 28 and $50 \mathrm{hpf}$ were larger than those between 50 and $72 \mathrm{hpf}$.

When olfactory sensory neurons of the transgenic fish were labeled with DiI, the signals in the olfactory placode and olfactory bulb merged well with those of EGFP (Fig. 3), suggesting that the $2.7 \mathrm{~kb}$ upstream sequence of the omp gene directed the expression in essentially all of the olfactory sensory neurons projecting to the olfactory bulb. Notably, axons converging to the olfactory plexus were DiI-positive and EGFP-negative.

\section{Effects of constitutively active and dominant-negative forms of PKA on axonal pathfinding of olfactory sensory neurons}

To examine the role of intracellular PKA signaling in the axon pathfinding of the olfactory neurons during development, we designed double-cassette vectors that direct the expression of two genes in the same olfactory neuron (Fig. 4A). Simple coinjection of two expression vectors resulted in a rare coexpression of the two genes. The DsRed-GFP double-reporter construct carried the $2.7 \mathrm{~kb}$ omp promoter-driven tau-DsRed fusion protein gene and the $0.7 \mathrm{~kb}$ omp promoter-driven tau-EGFP fusion protein gene linked in a tail-to-tail manner. We injected the doublereporter cassette vector into zebrafish embryos and examined the expression of two reporter genes by confocal microscopy. As shown in Figure $4 B$, tau-DsRed-positive olfactory neurons also expressed tau-EGFP (43 of 44 neurons in 12 embryos) and vice versa (43 of 46 neurons in 12 embryos), suggesting that the $2.7 \mathrm{~kb}$ and $0.7 \mathrm{~kb}$ promoters directed the expression in the same population of the cells. Thus, the double-cassette strategy was effective in directing the expression of two genes in the same cell of living zebrafish.

The PKA*-GFP construct carried the $2.7 \mathrm{~kb}$ omp promoterdriven constitutively active form of the mouse PKA $\alpha$ catalytic subunit gene (Orellana and McKnight, 1992) and the $0.7 \mathrm{~kb} o m p$ promoter-driven tau-EGFP fusion protein gene (Fig. $4 A$ ). Thus, PKA* $^{*}$ as an effector and tau-EGFP as a reporter should be expressed in the same olfactory neurons. Injection of the PKA* GFP cassette vector into zebrafish embryos labeled several olfactory neurons (Fig. 4D), which made it possible to follow the neurite extension of individual neurons during development. Zebrafish embryos injected with the GFP construct (Fig. $4 A$ ) served as a control (Fig. 4C). At $50 \mathrm{hpf}$, most of the olfactory neuron axons in control embryos were on their way to the target glomerulus in the olfactory bulb. Olfactory neuron axons in the embryos injected with the PKA*-GFP vector apparently extended further than those in the embryos injected with the control GFP vector (Fig. 4C,D). Several axons of the olfactory neurons reached the anterior-dorsal surface of the olfactory bulb in PKA*-injected embryos. Furthermore, axon trajectories of olfactory neurons in

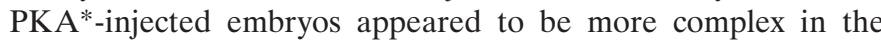
olfactory bulb near the BPB.

We also examined the effect of the suppression of PKA activity on the development of olfactory neurons. The dominant-negative form of the mouse type I regulatory subunit of PKA (Clegg et al., 1987) was placed in the effector-reporter cassette (Fig. 4A). In zebrafish embryos injected with the dnPKA-GFP vector, the axon trajectories of olfactory neurons seemed to be more complex in the olfactory placode at $50 \mathrm{hpf}$ (Fig. $4 E$ ). In addition, the axons of some olfactory neurons remained in the olfactory placode. The arrangement of olfactory neurons in dnPKA-injected embryos was disturbed.

\section{Region-specific effects of enhancement and suppression of PKA activity on axonal pathfinding}

To quantitatively assess the effects of the enhancement and suppression of PKA activity on how far the olfactory neurons reach their axon in the olfactory placode and the olfactory bulb, we 

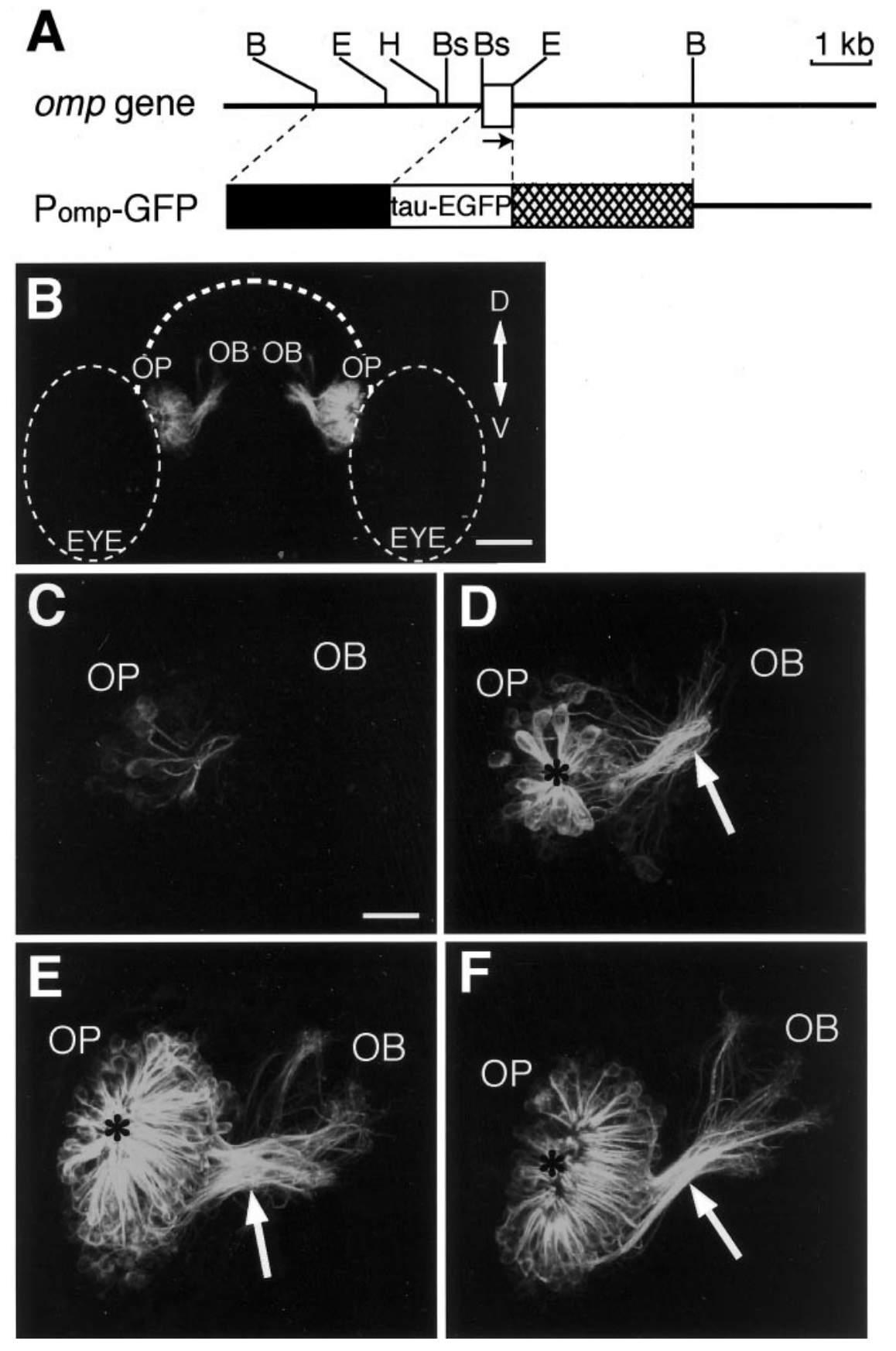

Figure 2. Axon extension of olfactory sensory neurons in living zebrafish carrying the omp promoterdriven tau-EGFP transgene. $A$, Genomic structure and restriction map of the zebrafish omp gene (top) and the structure of the omp promoter-driven tau-EGFP expression vector Pomp-GFP (bottom). A box indicates the coding region of the zebrafish omp gene. The arrow shows the direction of the transcription. $B, B s, E$, and $H$ represent the restriction sites for $B g l \mathrm{II}$, BspLU11I, Eco RI, and HindIII, respectively. The line of Pomp-GFP vector indicates the sequence of the pBluescript II SK+. The $2.7 \mathrm{~kb} B g l \mathrm{II}-B s p L U 11 \mathrm{I}$ upstream sequence and the $3.0 \mathrm{~kb} E c o$ RI-BglII downstream sequence of the $o m p$ gene were used to drive tau-EGFP expression. $B$, Anterior view of the head of a transgenic zebrafish embryo at $72 \mathrm{hpf}$. The composite image is generated from the series of optical sections and oriented to correspond to the composite images shown in $C-F$. Dorsal $(D)$ is to the top; ventral $(V)$ is to the bottom. $O B$, Olfactory bulb; $O P$, olfactory placode; $E Y E$, eye. Dashed lines indicate the outlines of the forebrain and the eyes. Scale bar, $60 \mu \mathrm{m}$. $C-F$, Axon pathfinding of developing olfactory sensory neurons in the right olfactory organ of living transgenic zebrafish embryos at $22(C), 28(D), 50(E)$, and 72 $(F)$ hpf. Dorsal is to the top, and medial is to the right. The anteroposterior axis is projecting out of the image. Arrows indicate the BPB, and asterisks indicate the nasal pit. $O B$, Olfactory bulb; $O P$, olfactory placode. Scale bar, $20 \mu \mathrm{m}$. measured the terminal reach and maximal reach values. We dotted at flections on the axonal process of a GFP-labeled olfactory neuron from the axonal hillock to the terminal in the reconstructed three-dimensional image. Each point was connected to form a trajectory of the axon. We set the origin of the measurements at the nasal pit, where the dendrites of olfactory sensory neurons converge, and measured distances from the origin to the axon terminal (terminal reach) and to the farthest point on the axon of interest (maximal reach). Both the terminal and maximal reach values of olfactory neuron axons were significantly larger in the $\mathrm{PKA}^{*}$-injected embryos at $50 \mathrm{hpf}$ than those in the control embryos (Fig. 5A,B) ( $t$ test; $p=0.004$ and 0.002 , respectively). There were no significant differences in these values for the axon reach between the dnPKA-injected and control embryos at $50 \mathrm{hpf}$ ( $t$ test; $p=0.16$ and 0.95 , respectively). However, 5 of 33 olfactory neuron axons of dnPKA-injected embryos had maximal reach values of $<40 \mu \mathrm{m}$ and remained within the olfactory placode region. In contrast, the maximal reach values of all 44 olfactory neuron axons in the $\mathrm{PKA}^{*}$-injected embryos and 33 olfactory neuron axons in the control embryos were $>48 \mu \mathrm{m}$, and these axons extended into the olfactory bulb beyond the BPB.

For quantitative assessment of the axonal turning, we introduced a parameter, complexity, which denotes the turning angle per $1 \mu \mathrm{m}$ length of axon and becomes larger as the number and angle of axon turns increase. There were no significant differences in the complexity of olfactory neuron axons among the control, PKA*-injected, and dnPKA-injected embryos at 50 hpf (Fig. 5C). However, we noticed that, in some specific regions, olfactory neurons in the $\mathrm{PKA}^{*}$ - and dnPKA-injected embryos appeared to show more complex turning of axons than those in the control 
Figure 3. DiI labeling of olfactory sensory neurons in a tau-EGFP transgenic zebrafish embryo at $84 \mathrm{hpf}$. Anterior-view images of a confocal section through the olfactory organ are shown. Tau-EGFP-expressing cells were visualized with an argon laser (left), and DiI-labeled cells were monitored with a helium-neon laser (middle). These images are merged on the right. An iris of the confocal microscopy was set to obtain fluorescent signals from the confocal plane with $\sim 4 \mu \mathrm{m}$ width. $O B$, Olfactory bulb; $O P$, olfactory placode. Scale bar, $10 \mu \mathrm{m}$.
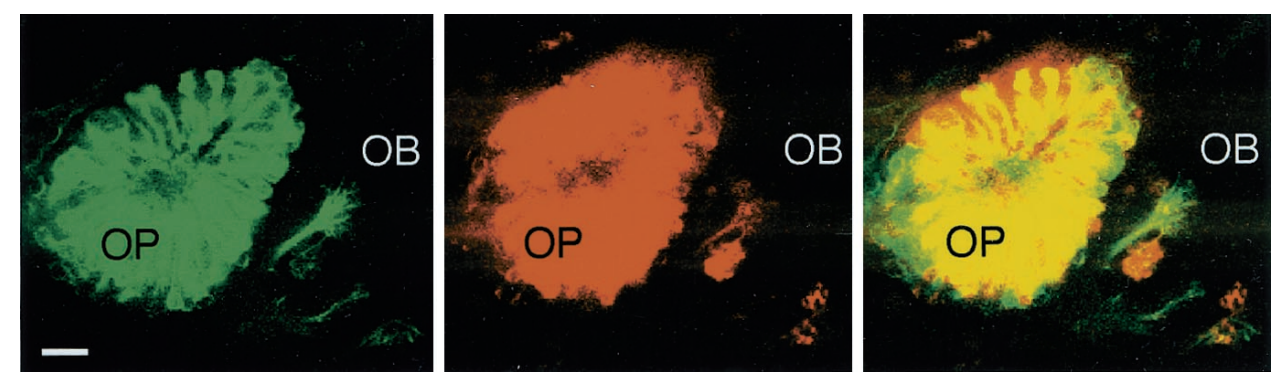

A
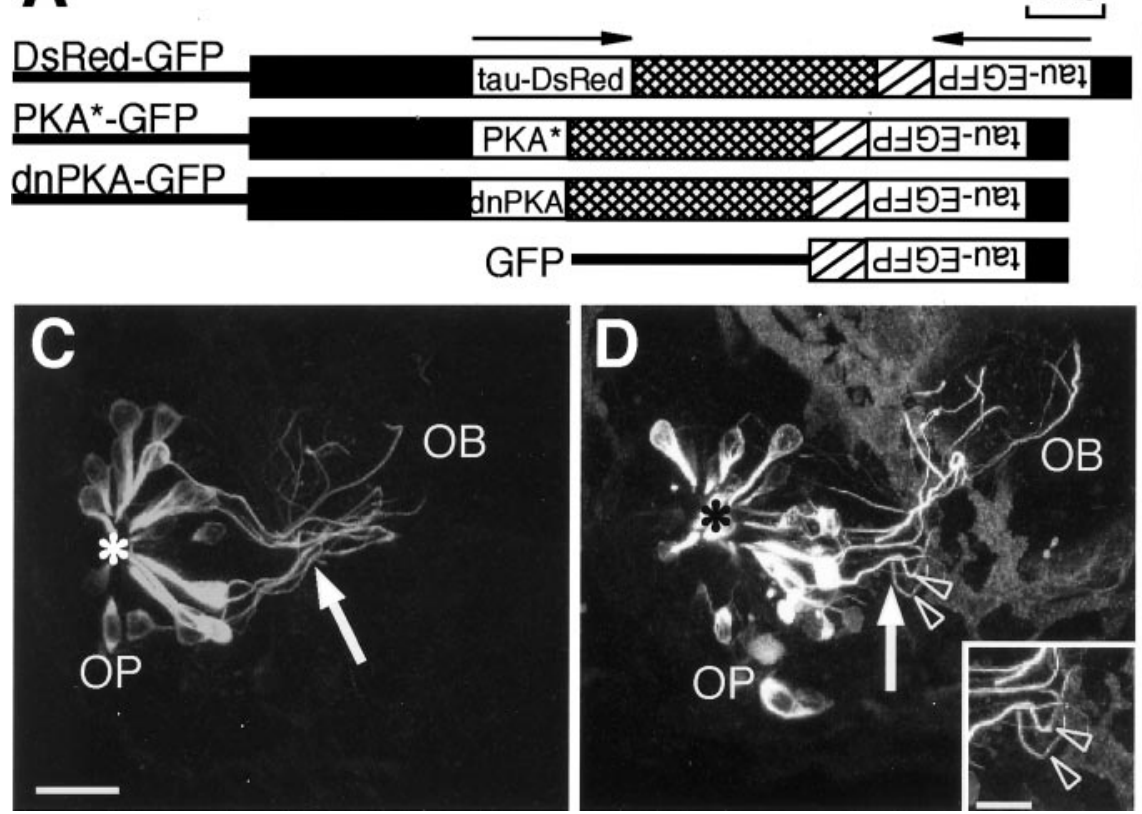

B
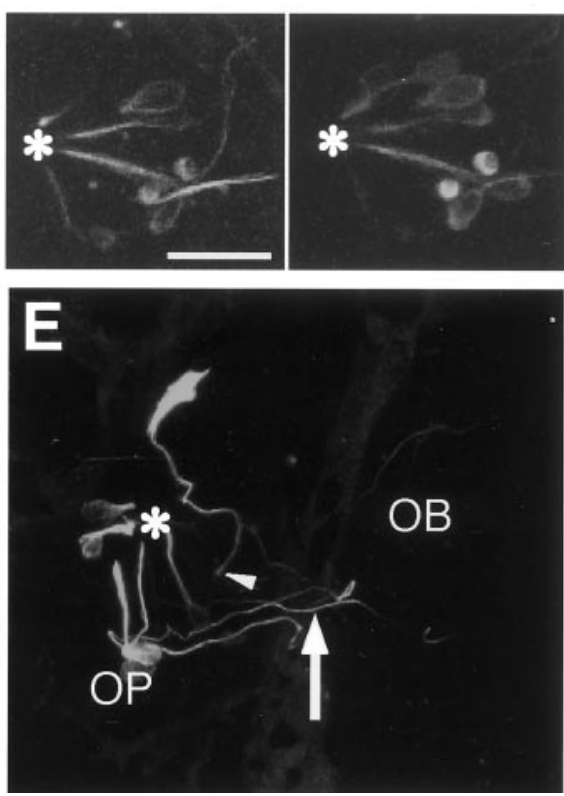

Figure 4. Effects of constitutively active and dominant-negative PKAs on the axon pathfinding of olfactory sensory neurons in living zebrafish embryos at $50 \mathrm{hpf}$. $A$, The structures of $o m p$ promoter-driven double-cassette expression vectors. Arrows indicate the direction of the transcription for each cassette. DsRed-GFP vector (top) carries two reporter genes. PKA*-GFP vector (second row) directs the expression of the constitutively active form of $\alpha$ catalytic subunit of PKA and tau-EGFP reporter gene. dnPKA-GFP vector (third row) carries the dominant-negative PKA regulatory subunit and tau-EGFP reporter gene. GFP vector (bottom) serves as a control. Black boxes indicate the 2.7 or $0.7 \mathrm{~kb} 5^{\prime}$ upstream sequence of the omp gene, crosshatched boxes indicate the $3.0 \mathrm{~kb} \mathrm{3'}$ downstream sequence of the omp gene, hatched boxes indicate the SV40 poly(A) additional sequence, and lines indicate pBluescript II SK+. B, Coexpression of tau-DsRed and tau-EGFP in olfactory sensory neurons of the zebrafish embryos injected with DsRed-GFP vector. An anterior view of the composite images of the right olfactory placode of an embryo at 50 hpf is shown. Tau-EGFP expression was visualized with an argon laser (left), and tau-DsRed expression was monitored with a helium-neon laser (right). An asterisk indicates the nasal pit. Scale bar, $20 \mu \mathrm{m}$. $C-E$, Axon pathfinding of developing olfactory neurons in embryos at $50 \mathrm{hpf}$. Zebrafish embryos were injected with GFP $(C)$, PKA*-GFP $(D)$, and dnPKA-GFP $(E)$ expression vectors. Arrows indicate the BPB, and asterisks indicate the nasal pit (origin). Arrowheads in D indicate the abnormal turning of the axonal trajectories. The arrowhead in $E$ points to the axon terminal that has not yet entered the olfactory bulb at 50 hpf. Scale bar, $20 \mu \mathrm{m}$. The inset in $D$ details typical wandering of PKA*-expressing axons. $O B$, Olfactory bulb; $O P$, olfactory placode. Scale bar, $10 \mu \mathrm{m}$.

embryos (Fig. 4D,E). We then subdivided the axon trajectory field into areas of $20 \mu \mathrm{m}$ width based on the distance from the nasal pit and measured the regional complexity and the angle and number of axon turns (Fig. 6A). The PKA*-injected embryos at $50 \mathrm{hpf}$ tended to show high complexity of olfactory neuron axons in the region $40-60 \mu \mathrm{m}$ from the nasal pit (Fig. 6B) $(t$ test; $p=$ $0.05)$. The angle of axon turns in the region $40-60 \mu \mathrm{m}$ from the nasal pit was significantly larger in the $\mathrm{PKA}^{*}$-injected embryos than in the control embryos (Fig. 6C) ( $t$ test; $p=0.04$ ). The number of axon turns with angles sharper than $30^{\circ}$ in this region was significantly larger in the $\mathrm{PKA}^{*}$-injected embryos than in the control embryos (Fig. 6D) ( $t$ test; $p=0.02$ ).

In the dnPKA-injected embryos at $50 \mathrm{hpf}$, the regional complexity of olfactory neuron axons was significantly higher in the region $20-40 \mu \mathrm{m}$ from the nasal pit than that in the control embryos (Fig. 6B) ( $t$ test; $p=0.04$ ). The angle of axon turns in this region was significantly larger in the dnPKA-injected embryos than in the control embryos (Fig. $6 C$ ) ( $t$ test; $p=0.006)$. The number of turns with angles sharper than $30^{\circ}$ in the region 20-40 $\mu \mathrm{m}$ from the nasal pit was significantly larger in the dnPKA-injected embryos than in the control embryos (Fig. 6D) $(t$ test; $p=0.006$ ).

The olfactory neuron axons fasciculated to form the olfactory nerve and crossed the BPB through a restricted region as described by Hansen and Zeiske (1993). To determine the position of the BPB, we used data from embryos of the tau-EGFP line. The distance from the origin to the position at which olfactory neuron axons started to fasciculate was $38.3 \pm 1.4 \mu \mathrm{m}$ (mean \pm SEM; $n=7$ ), and that from the origin to the position at which olfactory neuron axons started to defasciculate was $46.6 \pm 1.5 \mu \mathrm{m}$ 

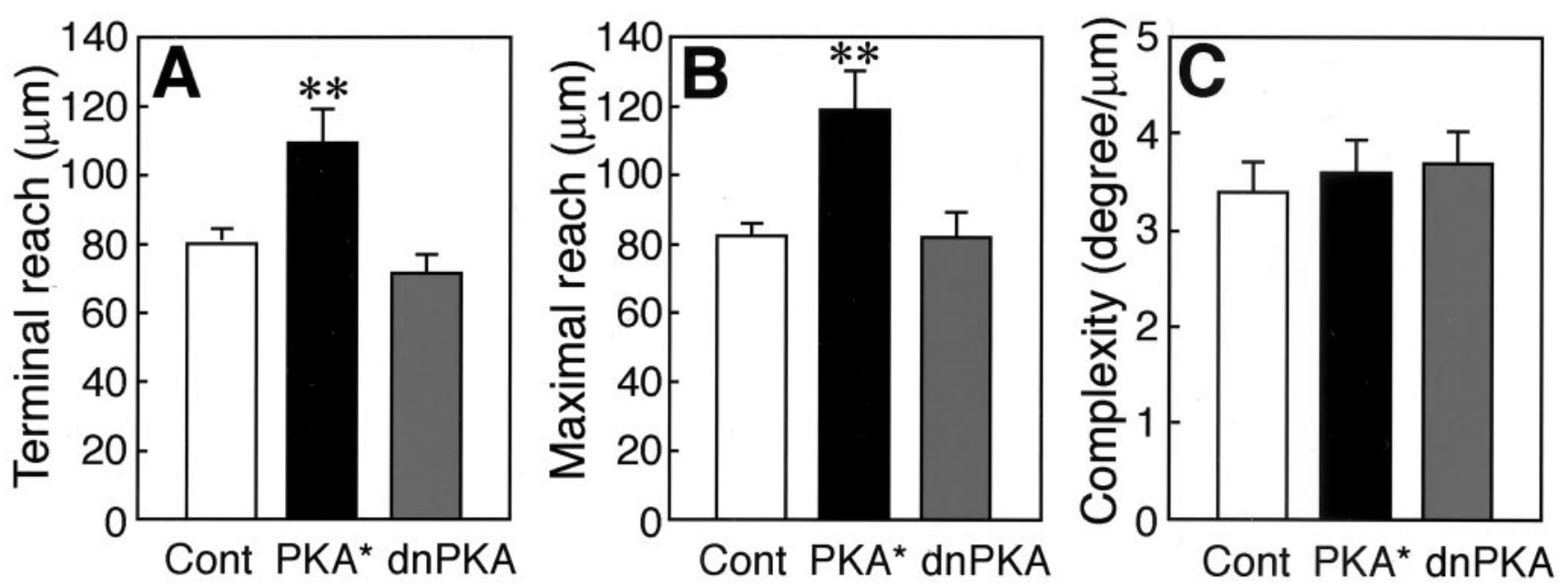

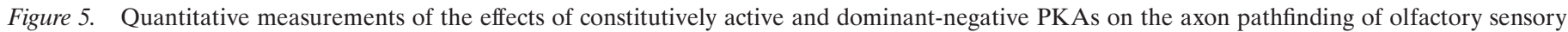

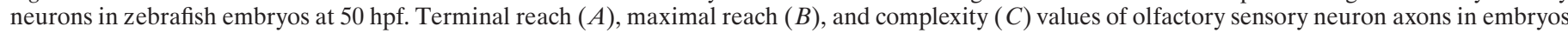

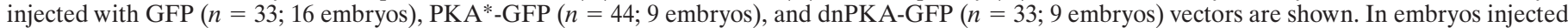

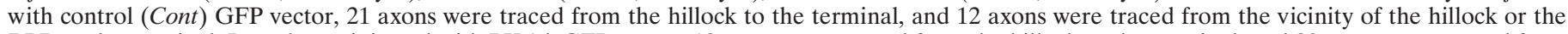

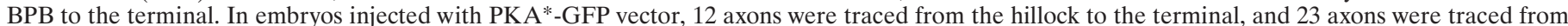

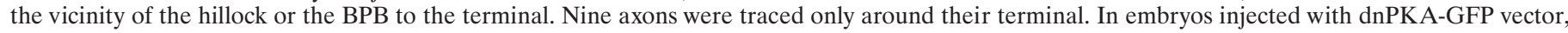

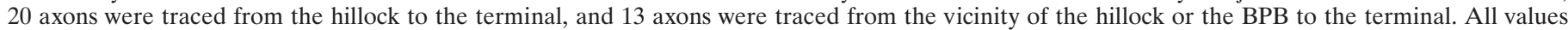
represent mean $\pm \mathrm{SEM} ; * p<0.01$.

$(n=7)$. We estimated that the BPB was positioned $\sim 40 \mu \mathrm{m}$ from the origin at $50 \mathrm{hpf}$. Thus, the dominant-negative form of PKA showed a clear effect in the olfactory placode, whereas the effect of the constitutively active form was prominent in the olfactory bulb immediately beyond BPB.

\section{Selective effect of forskolin treatment on axonal pathfinding in the olfactory bulb}

We then examined the effect of forskolin, an activator of adenylyl cyclase, on the axonal pathfinding of olfactory sensory neurons. Barresi et al. (2000) reported that zebrafish embryos treated with $300 \mu \mathrm{M}$ forskolin from $5.5 \mathrm{hpf}$ showed drastic morphological effects. We thus treated the embryos of tau-EGFP transgenic lines with $50 \mu \mathrm{M}$ forskolin from $18 \mathrm{hpf}$ and followed the axonal trajectories of olfactory sensory neurons at 22, 28, and $40 \mathrm{hpf}$ (Fig. $7 A-F)$. Under these conditions, there were no gross morphological abnormalities in the heads of the embryos at $40 \mathrm{hpf}$ (Fig. $7 G, H)$, although the treated embryos had a slight curve dorsally and had short tails. The olfactory neuron axons of both the forskolin- and mock-treated embryos equally extended within the olfactory placode and entered into the olfactory bulb at $\sim 22-28$ hpf (Fig. 7A-D). In the mock-treated control embryos, olfactory neuron axons stretched out in the olfactory bulb at $40 \mathrm{hpf}$ (Fig. $7 E$ ). In contrast, the olfactory neuron axons in the forskolintreated embryos retarded in the olfactory bulb near the BPB (Fig. $7 F$ ). Thus, the pharmacological manipulation for enhancing PKA signaling disturbed the axonal pathfinding of olfactory sensory neurons selectively in the olfactory bulb immediately after passing the BPB.

\section{DISCUSSION}

Selective labeling with the omp promoter-driven tau-EGFP enabled visualization of the development of axons of entire olfactory sensory neurons in transparent living zebrafish embryos. Furthermore, we constructed double-cassette vectors, in which the omp promoter-driven dominant forms of PKA and marker protein tau-EGFP genes were physically linked, and microin- jected them into zebrafish eggs to visualize and manipulate the olfactory sensory neurons simultaneously. This novel strategy made it possible to label a few olfactory neurons and examine the effect of alterations in PKA signaling on the pathfinding of their axons in vivo. Although the expression levels of the dominant forms of PKA would be variable among the injected olfactory neurons, we observed clear effects of such manipulations on axonal pathfinding in vivo.

The expression of a constitutively active form of PKA in zebrafish olfactory neurons significantly increased the terminal and maximal reach values at $50 \mathrm{hpf}$. However, at $30 \mathrm{hpf}$, when most of the olfactory neuron axons have already extended their terminals into the olfactory bulb, the net axon lengths measured from the axon hillock to the terminal were comparable among the

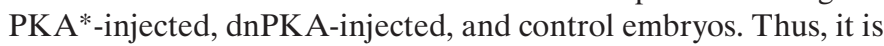
likely that the enhancement of PKA activity hardly affected the speed of axon extension in the olfactory placode. Manipulation of PKA signaling in the olfactory neurons did not appreciably affect axon branching. The terminal reach values of the PKA*-injected embryos at $50 \mathrm{hpf}$ were larger than the maximum terminal reach values of transgenic tau-EGFP lines at $50 \mathrm{hpf}(125 \pm 3.5 \mu \mathrm{m})$ and $72 \mathrm{hpf}(103 \pm 2.3 \mu \mathrm{m})$, which never exceeded $153 \mu \mathrm{m}$. Nine of 44 olfactory neuron axons of the PKA*-injected embryos at $50 \mathrm{hpf}$ had terminal reach values of $>160 \mu \mathrm{m}$. Because olfactory neurons project their axons to restricted targets in the olfactory bulb and form glomerular-like terminal structures within 2-3 d of development (Wilson et al., 1990; Dynes and Ngai, 1998), the constitutively active form of PKA may disturb the axon termination at the glomerulus.

We found that the expression of a constitutively active form of PKA significantly increased the turning of axons in the region $40-60 \mu \mathrm{m}$ from the nasal pit. This region was in the olfactory bulb near the BPB. The expression of a dominant-negative PKA regulatory subunit also affected the turning of axons, but in the region $20-40 \mu \mathrm{m}$ from the nasal pit. The region where the turning of olfactory neuron axons was sensitive to the dominant-negative 

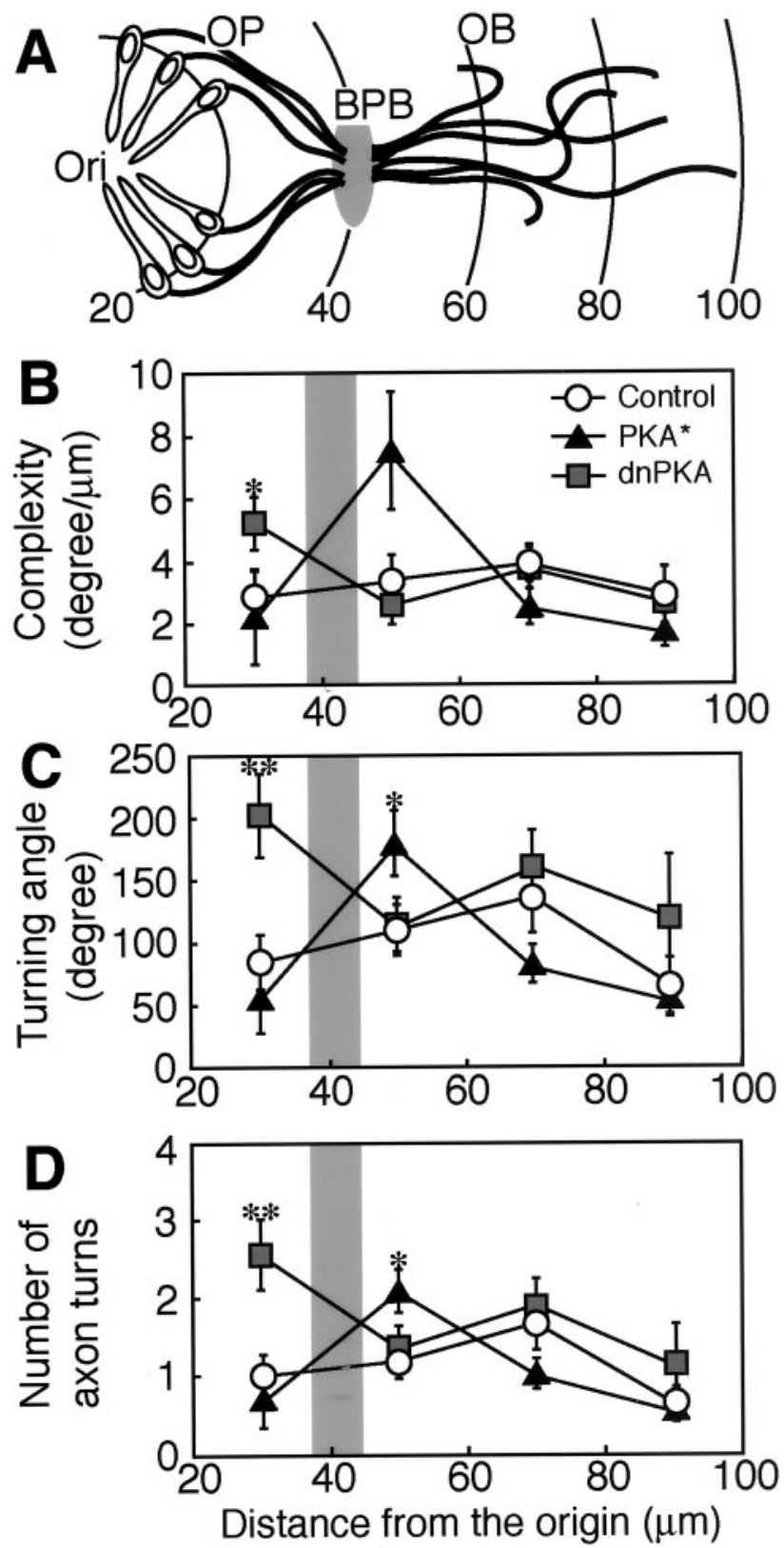

Figure 6. Regional differences in the effects of constitutively active and dominant-negative PKAs on the turning of olfactory sensory neurons in zebrafish embryos at $50 \mathrm{hpf}$. $A$, A schematized model for the position of the olfactory placode $(O P)$, the olfactory bulb $(O B)$, and their boundary $(B P B)$. The axon trajectory field was subdivided into areas of $20 \mu \mathrm{m}$ width based on the distance from the nasal pit. Arches and values indicate the distance from the nasal pit (Ori) in micrometers. $B$, Regional complexity of axons. Complexity value was calculated by dividing the summation of turning angles in each area by the summation of the axon length within the area. $C$, Turning angles of axons. The summation of turning angles in each area is compared. $D$, Number of axon turns. The numbers of flections with the angle larger than $30^{\circ}$ in each area are compared. All values represent mean \pm SEM. Shaded regions in $B-D$ indicate the position of the BPB. The numbers of axons analyzed in the areas of $20-40,40-60,60-80$, and $80-100 \mu \mathrm{m}$ were $17,33,33$, and 17 , respectively, for embryos injected with control GFP vector; 9, 30, 30, and 17, respectively, for embryos injected with PKA*-GFP vector; and 20, 28, 27, and 14 , respectively, for embryos injected with dnPKA-GFP vector. ${ }^{*} p<$ $0.05 ;{ }^{* *} p<0.01$

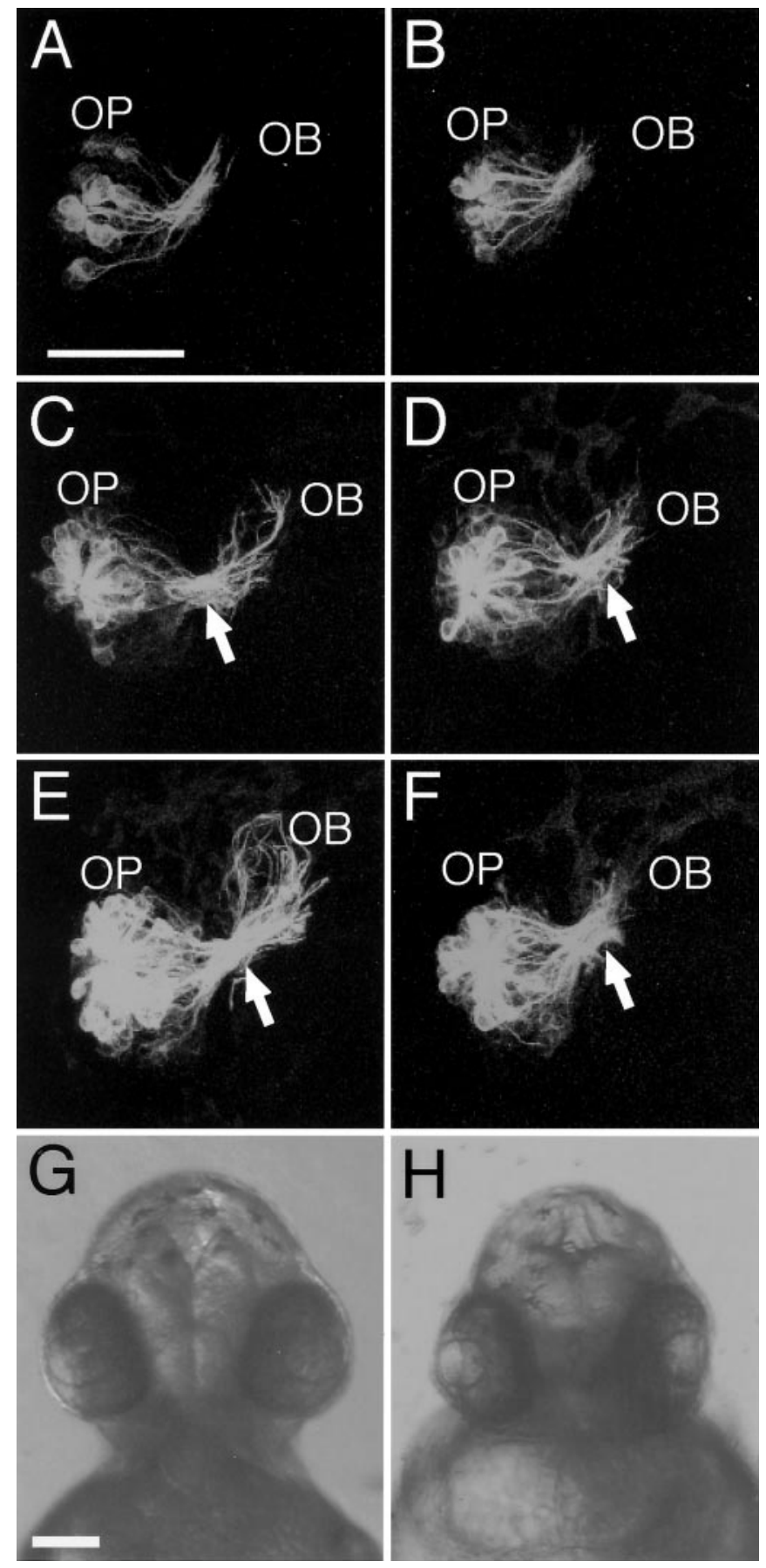

Figure 7. Effects of forskolin treatment on the axon pathfinding of olfactory sensory neurons in tau-EGFP transgenic zebrafish embryos. $A-F$, Representative anterior-view images of the olfactory neuron axon trajectories in the right olfactory organ of $50 \mu \mathrm{M}$ forskolin-treated embryos $(B, D, F)$ and mock-treated control embryos $(A, C, E)$ at $22(A, B)$, $28(C, D)$, and $40(E, F)$ hpf. Dorsal is to the top. $O B$, Olfactory bulb; $O P$, olfactory placode. Arrows point to the BPB. Scale bar, $50 \mu \mathrm{m}$. $G, H$, Anterior views of mock-treated $(G)$ and forskolin-treated $(H)$ embryos at $40 \mathrm{hpf}$. Scale bar, $100 \mu \mathrm{m}$.

PKA was in the olfactory placode. These results show that the switching from the dominant-negative PKA-sensitive state to the constitutively active PKA-sensitive state takes place at a region $\sim 40 \mu \mathrm{m}$ from the nasal pit, which corresponds to the BPB. Suppression of PKA activity by the expression of the dominant- 


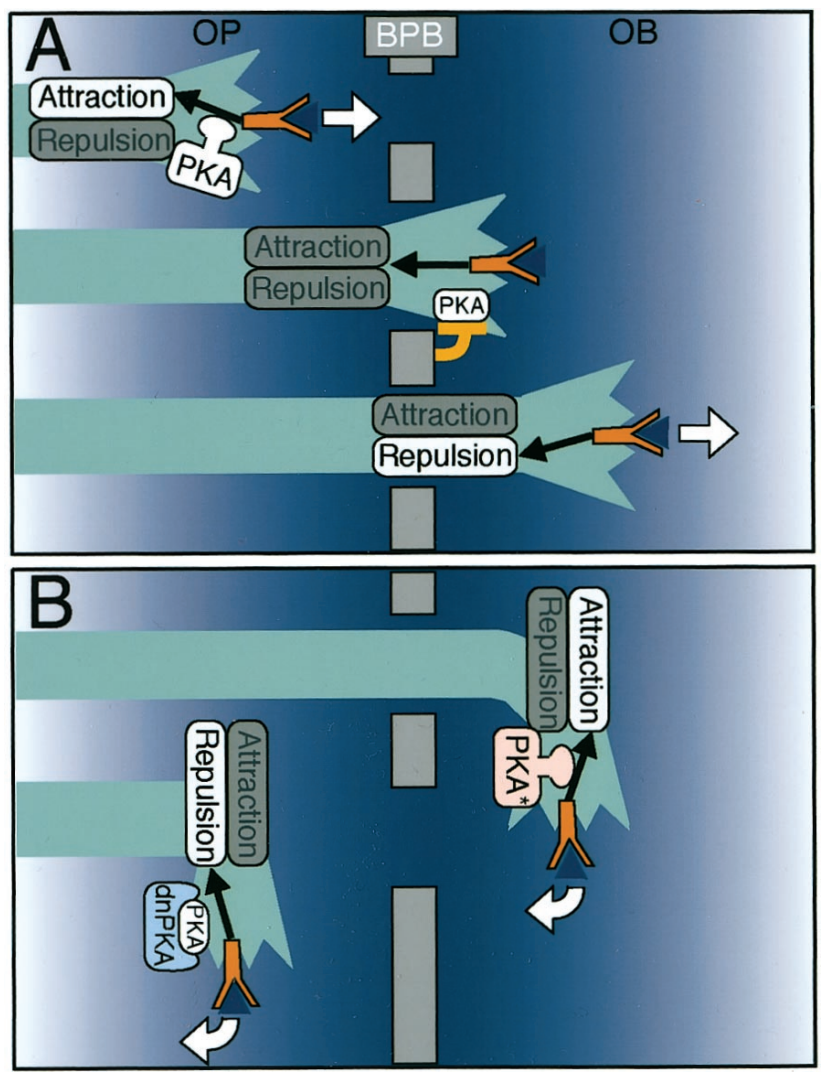

Figure 8. A hypothetical model for the regulatory role of PKA in the axon extension of olfactory sensory neurons through the BPB in zebrafish. $A$, In this model, we propose that PKA activity would modulate intracellular guidance signals (black arrows) from receptors (red Y-shaped figures) on growing olfactory axons to show attractive responses to presumptive guidance molecules toward the BPB (blue triangles). When olfactory neuron axons reach the $\mathrm{BPB}$, the interaction of growing axons and the BPB would downregulate PKA. Loss or decrease of PKA activity would change the sensitivity of the olfactory neurons to guidance molecules from attractive to repulsive, making it possible for growing axons to leave the $\mathrm{BPB}$ rather than turning back toward it. $O B$, Olfactory bulb; $O P$, olfactory placode. White arrows indicate the direction of axon extension. $B$, According to the model, expression of the constitutively active PKA (PKA*) in the olfactory sensory neurons would make the guidance molecule signal attractive even in the olfactory bulb, leading to a disturbance of the direction of axon extension in the olfactory bulb. In contrast, expression of dnPKA would make the guidance molecule signals in the olfactory placode repulsive, thus disturbing the direction of the axon extension in the olfactory placode.

negative regulatory subunit enhanced the axon turning of olfactory sensory neurons in the olfactory placode. Furthermore, $\sim 15 \%$ of the olfactory neurons injected with dnPKA failed to send out their axons into the olfactory bulb through the BPB, and their axon terminals remained in the olfactory placode. The prominent effect of the dominant-negative PKA but not of the constitutively active PKA suggests that PKA signaling is active in the olfactory placode and important for correct pathfinding toward the olfactory bulb through the BPB. The effect of the dominant-negative PKA disappeared in the olfactory bulb even in the region close to the BPB. Rather, the constitutively active PKA clearly affected the turning of developing axons in the olfactory bulb in the vicinity of the BPB. The disturbance of the axonal pathfinding by the gain of PKA function but not by the loss of PKA function suggests that, in contrast to the olfactory placode, PKA signaling is less active in the region of the olfactory bulb close to the BPB. Consistently, the enhancement of PKA signaling by forskolin treatment exerted retardatory effects on the axonal pathfinding in the olfactory bulb near the BPB, but not in the olfactory placode. Our results thus suggest that PKA signaling is altered from the active state to the inactive state in the developing olfactory neurons, and that the switching of PKA signaling is important for axonal pathfinding of developing olfactory sensory neurons through the BPB in vivo.

The developing axons of olfactory sensory neurons crossed the $\mathrm{BPB}$ through a restricted region and branched out to several zones in the olfactory bulb. The dominant-negative PKA interfered with the extension of the sensory neuron axons toward the $\mathrm{BPB}$, whereas the constitutively active PKA disturbed the extension from the BPB. Interestingly, in vitro studies showed that high levels of intracellular cAMP directed the attractive turning of cultured Xenopus spinal neurons to Netrin or BDNF, and low levels directed repulsive turning (Ming et al., 1997; Song et al., 1997). Thus, it seems reasonable that the switching of PKA signaling modulates the sensitivities of the growing axons to presumptive guidance cues toward BPB as an intermediate guidepost. Active PKA signaling in the olfactory placode would be important for growing axons to show an attractive response to the guidance molecules. We propose that the interaction of the extending olfactory neuron and BPB may downregulate PKA signaling in the olfactory neuron, and that the reduction in the extent of PKA signaling would alter the response of the growth cone to the BPB from attractive to repulsive (Fig. 8). Although attractive and repulsive guidance molecules play key roles in axonal pathfinding (Tessier-Lavigne and Goodman, 1996; Mueller, 1999), there should be an ingenious regulatory mechanism for the responsiveness of growing axon terminals toward guidance cues for long-distance axon guidance, where extending axons once attracted toward intermediate guideposts have to leave them. Plastic modulation of the axonal responses by PKA switching may underlie the long-distance axon guidance by intermediate guideposts.

\section{REFERENCES}

Barresi MJF, Stickney HL, Devoto SH (2000) The zebrafish slowmuscle-omitted gene product is required for hedgehog signal transduction and the development of slow muscle identity. Development 127:2189-2199.

Brand A (1995) GFP in Drosophila. Trends Genet 11:324-325.

Buiakova OI, Krishna NS, Getchell TV, Margolis FL (1994) Human and rodent OMP genes: conservation of structural and regulatory motifs and cellular localization. Genomics 20:452-462.

Clegg CH, Correll LA, Cadd GG, McKnight GS (1987) Inhibition of intracellular cAMP-dependent protein kinase using mutant genes of the regulatory type I subunit. J Biol Chem 262:13111-13119.

Dynes JL, Ngai J (1998) Pathfinding of olfactory neuron axons to stereotyped glomerular targets revealed by dynamic imaging in living zebrafish embryos. Neuron 20:1081-1091.

Farbman AI, Margolis FL (1980) Olfactory marker protein during ontogeny: immunohistochemical localization. Dev Biol 74:205-215.

Hansen A, Zeiske E (1993) Development of the olfactory organ in the zebrafish, Brachydanio rerio. J Comp Neurol 333:289-300.

Harris R, Sabatelli LM, Seeger MA (1996) Guidance cues at the Drosophila CNS midline: identification and characterization of two Drosophila netrin/UNC-6 homologs. Neuron 17:217-228.

Hong K, Hinck L, Nishiyama M, Poo MM, Tessier-Lavigne M, Stein E (1999) A ligand-gated association between cytoplasmic domains of UNC5 and DCC family receptors converts netrin-induced growth cone attraction to repulsion. Cell 97:927-941.

Jowett T (1999) Analysis of protein and gene expression. Methods Cell Biol 59:63-85.

Kidd T, Brose K, Mitchell KJ, Fetter RD, Tessier-Lavigne M, Goodman CS, Tear G (1998) Roundabout controls axon crossing of the CNS midline and defines a novel subfamily of evolutionarily conserved guidance receptors. Cell 92:205-215.

Kidd T, Bland KS, Goodman CS (1999) Slit is the midline repellent for the robo receptor in Drosophila. Cell 96:785-794. 
Ming GL, Song HJ, Berninger B, Holt CE, Tessier-Lavigne M, Poo MM (1997) cAMP-dependent growth cone guidance by netrin-1. Neuron 19:1225-1235.

Mitchell KJ, Doyle JL, Serafini T, Kennedy TE, Tessier-Lavigne M, Goodman CS, Dickson BJ (1996) Genetic analysis of Netrin genes in Drosophila: netrins guide CNS commissural axons and peripheral motor axons. Neuron 17:203-215.

Mori H, Miyazaki Y, Morita T, Nitta H, Mishina M (1994) Different spatio-temporal expressions of three otx homeoprotein transcripts during zebrafish embryogenesis. Brain Res Mol Brain Res 27:221-231.

Mueller BK (1999) Growth cone guidance: first steps towards a deeper understanding. Annu Rev Neurosci 22:351-388.

Orellana SA, McKnight GS (1992) Mutations in the catalytic subunit of cAMP-dependent protein kinase result in unregulated biological activity. Proc Natl Acad Sci USA 89:4726-4730.

Rössler P, Mezler M, Breer H (1998) Two olfactory marker proteins in Xenopus laevis. J Comp Neurol 395:273-280.
Shirasaki R, Katsumata R, Murakami F (1998) Change in chemoattractant responsiveness of developing axons at an intermediate target. Science 279:105-107.

Song HJ, Ming GL, Poo MM (1997) cAMP-induced switching in turning direction of nerve growth cones. Nature 388:275-279.

Stein E, Tessier-Lavigne M (2001) Hierarchical organization of guidance receptors: silencing of netrin attraction by slit through a robo/ DCC receptor complex. Science 291:1928-1938.

Tessier-Lavigne M, Goodman CS (1996) The molecular biology of axon guidance. Science 274:1123-1133.

Whitlock KE, Westerfield M (1998) A transient population of neurons pioneers the olfactory pathway in the zebrafish. J Neurosci 18:8919-8927.

Wilson SW, Ross LS, Parrett T, Easter SSJ (1990) The development of a simple scaffold of axon tracts in the brain of the embryonic zebrafish, Brachydanio rerio. Development 108:121-145. 